\title{
Magnetic survey of bright northern main sequence stars ${ }^{\star}$
}

\author{
D. N. Monin, S. N. Fabrika, and G. G. Valyavin
}

\begin{abstract}
Special Astrophysical Observatory of Russian AS, Nizhnij Arkhyz 369167, Russia
Received 26 June 2000 / Accepted 30 August 2002

Abstract. The first results of a systematic search for magnetic fields in the brightest upper main sequence (MS) stars are presented. The main goal is to survey the stars with about the same detection limit and to improve existing statistics of their magnetism. The target list contains 57 upper MS stars and represents well B0.5-F9 stars. High-resolution Zeeman spectra were obtained for 30 stars of the list. The accuracy of the magnetic field measurements ranges from 20 to $300 \mathrm{G}$ depending mainly on spectral class. In the majority of studied stars we did not detect magnetic fields. In some stars we suspect the presence of a weak magnetic field. These are the best candidates for more extensive studies. A particular case is the star $\chi$ Dra where we probably detected the global magnetic field. The longitudinal field strength is $B_{1}=-54 \pm 12 \mathrm{G}$. Further observations of this star are needed to confirm the detection and to ascertain if the magnetic field is variable with the period of rotation.
\end{abstract}

Key words. stars: magnetic fields - surveys

\section{Introduction}

Magnetic fields have been discovered in many types of stars having different evolutionary status: pre-MS stars, MS stars, subdwarfs and white dwarfs. Often they are definitely and repeatedly detected in chemically peculiar (CP) MS stars as well. Longitudinal magnetic fields of MS stars range from $10^{2}$ to $10^{4} \mathrm{G}$. Some white dwarfs show strong magnetic fields in the range $10^{4}-10^{9} \mathrm{G}$ (Schmidt \& Smith 1995; McCook \& Sion 1999; Fabrika \& Valyavin 1999). There is evidence that the field evolves during a star's life. It seems to appear when a star completes a significant part of its MS lifetime (Hubrig et al. 2000), and the field strength changes across the MS (Glagolevskij 1987). Valyavin \& Fabrika (1999) found that the frequency of magnetic white dwarfs begins with $2 \%$ among hot (young) degenerate stars and reaches $20 \%$ among cool ones. This means that their initial fields are amplified with the time. However the nature of these initial fields is still unclear. The field in white dwarfs can be produced in the process of collapse of their progenitors, MS stars which possess surface fields of $1-10^{4} \mathrm{G}$, by simple flux conservation. This hypothesis is based mainly on the coincidence of magnetic fluxes. To study this subject the general magnetic properties of both MS stars and white dwarfs should be analyzed and compared.

The first statistical investigation of magnetic fields in white dwarfs was done by Angel et al. (1981). Their method has been improved by Fabrika et al. (1997) and Fabrika \& Valyavin (1999). They introduced the magnetic field function (MFF) - a normalized distribution of the star's

\footnotetext{
Send offprint requests to: D. N. Monin,

e-mail: mosha@sao.ru

* Based on observations collected at the $1 \mathrm{~m}$ telescope of the Special Astrophysical Observatory (Nizhnij Arkhyz, Russia).
}

frequencies $P_{\mathrm{B}}$ versus magnetic field strength calculated per one Gauss. Now we have a powerful tool to study the incidence of magnetism in MS stars.

We need to know how frequent magnetic stars are, how many of them are weakly magnetic, whether they are a peculiar class of stars or they are just a magnetic tail in a single distribution of magnetic fields. Magnetic fields and surface chemical peculiarities in Ap stars are known to be closely related, but the mechanism of this relation is unclear. It is still unknown what the minimum magnetic field strength is that can introduce the mechanism and how it depends on other parameters of the stars. In the study of general magnetic properties one may hope to find magnetic field evolution both on the MS (Hubrig et al. 2000), and after that in subsequent evolution to the white dwarf stage.

After the first observations by Babcock (1958), many authors have published results of their studies of magnetic fields in upper MS stars. These results have been compiled by Romanyuk (2000). The measurements are not homogeneous both in methods (see for review Landstreet 1980), accuracies and selection criteria. The magnetic field measurements have so far been made mainly in stars that are likely to possess magnetic fields, sharp lined CP stars. Furthermore, authors do not often publish null results. In the next section we estimate the incidence of magnetism among MS stars using the data available.

The most extensive study of normal MS stars made with high precision is by Landstreet (1982). He searched for longitudinal magnetic fields in $31 \mathrm{O}-\mathrm{F}$ upper MS stars and $5 \mathrm{Am}$ stars. No magnetic fields were detected with a median accuracy of about $65 \mathrm{G}$. Both a Balmer-line Zeeman analyzer and a two-channel coude line-profile scanner equipped with a polarization modulator were used in this study. Only 13 out of 31 stars were observed with the first device, both slow and fast 
rotating stars. The second device was applied to obtain heliumand iron-line measurements for slow rotating stars. Thus, the full sample cannot be considered to be unbiased.

To apply statistical methods one needs an unbiased (selection-free) sample of stars. It is obvious that the number of selection criteria must be diminished as much as possible. It seemed appropriate to undertake a systematic magnetic survey of upper MS stars. We started such a survey using the same observing method and data reduction for all samples. In this paper we report the first results. Special attention was paid to selection criteria. In Sect. 3 we describe the selection criteria, observational limitations, and the list of program stars. In Sect. 4 we present the steps of data reduction. Using the method of weighted coefficients along with the continuum reconstruction procedure we have obtained a high accuracy of magnetic field measurements.

\section{Magnetic field distributions of MS stars}

The first attempt to construct the surface magnetic field distribution of Ap stars was made by Preston (1971). Only extremely slowly rotating Ap stars were chosen in his study. Estimates of mean surface fields were obtained from measurements of magnetically resolved or broadened absorption lines. The distribution has a maximum between 2 and $3 \mathrm{kG}$ and a tail of stronger fields extended beyond $10 \mathrm{kG}$. The number of stars with a field below $2 \mathrm{kG}$ is decreased. The behavior below $2 \mathrm{kG}$ was explained by observational limits. In the low-field region the measurements become highly uncertain.

In their extensive study, Mathys et al. (1997) constructed the distribution of Ap stars using surface magnetic fields derived from measurements of magnetic splitting of the FeII $\lambda 6149.2$ line. This distribution has almost the same form as that obtained by Preston (1971). It has a maximum between 3 and $5 \mathrm{kG}$. Mathys et al. (1997) pointed out an insufficient number of stars with a field less than $3 \mathrm{kG}$. The lowest field found by them is $2.7 \mathrm{kG}$. This could not be explained by observational limitations only because the resolution limit corresponded to $1.7 \mathrm{kG}$.

It can be concluded from the analysis of both these distributions that the magnetic star frequency has a maximum at $3-5 \mathrm{kG}$ in the range of surface magnetic fields from a couple of $\mathrm{kG}$ to a few tens of $\mathrm{kG}$. Nevertheless, Bohlender \& Landstreet (1990a) and Landstreet (1992) have found a continuous distribution of Ap stars in rms longitudinal magnetic fields strongly skewed toward small field values down to the limit of detectability and a long high-field tail. Note that the last authors used estimates of longitudinal fields derived by another method, the Balmer-line Zeeman analyzer technique.

In our opinion such an inconsistency is not surprising because of the direct limitations and specific selection criteria. To enhance the probability of detection, targets are isolated using chemical peculiarities, narrow lines, etc. There is a relation between magnetic field strength and a chemical peculiarity value (North 1980; Cramer \& Maeder 1980). Up to the surface field $B_{\mathrm{S}} \approx 5 \mathrm{kG}$, stronger fields are accompanied by higher peculiarities. The stars with a spectral (and photometric) peculiarity are usually used as targets to find magnetic fields. The data on stars with strong fields is incomplete, but they are dramatically incomplete for stars having weak fields.

Nevertheless, we can roughly estimate the frequency of magnetic stars. There are 161 magnetic MS stars that are brighter than 7.5 and whose spectral types are in the range B5-F2 (Romanyuk 2000). We consider MS stars of luminosity class V only. According to the Hipparcos data there are 3236 B5-F2 MS stars brighter than 7m. 5. Therefore, at least 5\% of upper MS stars seem to be magnetic. It is obvious that this number is only a lower limit because not all 3236 stars have been studied for the presence of the field. On the other hand, Egret \& Jaschek (1981) estimated the frequency of CP stars using the catalogue of stellar groups. They have found that the fraction of Ap stars ( $\mathrm{Si}, \mathrm{Cr}, \mathrm{Eu}, \mathrm{Sr}$ and $\mathrm{Hg}-\mathrm{Mn}$ stars) is about $11 \%$ among B7-A3 stars. One may consider this number to be an upper limit of the frequency of magnetic stars because not every Ap star being observed for the presence of magnetic field is detected as magnetic.

Estimating the frequency of magnetic stars the question has to be answered: what is the magnetic field strength above which a star can be considered as magnetic? This limiting magnetic field value $B_{\text {lim }}$ depends directly on the current observational possibilities. By improving observational techniques and decreasing $B_{\text {lim }}$ we find magnetic stars to be more frequent. The MFF is free of this limitation because both positive and negative detections are used in estimations of frequencies (Fabrika et al. 1997). The first MFF for MS stars was presented by Bychkov et al. (1997). In logarithmic coordinates it has been found to be almost linear $\log \left(P_{\mathrm{B}}\right)=-\alpha \log \left(B_{\mathrm{S}}\right)+$ const. in the range of surface fields $\approx 3 \times 10^{3}-3 \times 10^{4} \mathrm{G}$, the spectral index is $\alpha \approx 1.9$. Fabrika \& Valyavin $(1998,1999)$ have found that the distribution of white dwarfs (the surface fields $\approx 10^{4}-10^{9} \mathrm{G}$ ) is also linear, but it has the spectral index $\alpha=1.3 \pm 0.1$. Both the distributions are represented well by a power law, but their spectral indices are quite different. This fact may be related to evolution effects. However, the difference in the spectral slopes may also be related to insufficient accuracy of the distribution of MS stars. Bychkov et al. (1997) noted that the distribution can have a different behavior in the low-field part where the surface field is less than a few kG. Furthermore, Bychkov et al. (1997) pointed out that constant term const. could not be determined correctly because the frequency of magnetic stars is not known well. The authors used only available published data. These data are greatly distorted by observational selection.

To investigate the magnetic properties of MS stars as a whole, it is necessary to use an unbiased sample of stars with a high accuracy of measurements and to cover as many stars as possible. To get better statistics, one should observe many stars, rather than to study some of them in detail. There is a probability of null detection of the effective magnetic field in a single observation because of stellar rotation. To take into account the probability of null detection, a missing factor should be introduced (Schmidt \& Smith 1995; Fabrika \& Valyavin 1999). The upper MS stars show large-scale, rather dipolar magnetic fields. In dipolar geometry the missing factor depends on the accuracy of measurements and the interval of effective 
magnetic fields considered. Therefore, it can be calculated. This allowed us to choose a "single observation" strategy.

\section{The list}

There are two obvious ways to select targets - volume limited and magnitude limited. The apparent density of hot stars is low compared to cool stars. The nearest early B-type MS star is $31 \mathrm{pc}$ from the Sun, and 108 F-type MS stars occupy this volume. Apart from great differences in sample sizes for cool and hot stars, the volume-limited selection also introduces differences in accuracy of magnetic field measurements. Because target magnitudes spread over a wide range, the difference of magnitudes can reach $9^{\mathrm{m}}$. This cannot be compensated by adjustment of exposure times.

Another way is to observe all stars up to the limiting stellar magnitude. It increases the apparent density of hot stars with respect to that of cool stars. Hot and cool stars are selected inside volumes that differ in size. This may introduce some bias, if the stars were not well mixed in space after their birth. They may have some from the common properties host.

The obvious observational selection which we cannot avoid in any selecting scheme is the different accuracy of magnetic field measurements in stars of different spectral classes. For fast rotating A-type stars with few measurable lines in their spectra the accuracy will be essentially worse. A natural way to solve the problem is to increase the number of stars involved in the study in order to test different spectral types separately. From the above arguments, the magnitude-limited selecting scheme seems more appropriate.

We formed our list using the Bright Star Catalogue (BSC, Hoffleit \& Jaschek 1982). The criteria for stars to be included in the sample are as follows:

- stellar magnitude $V \leq 4^{\mathrm{m}}$. 0 ,

- luminosity class $\mathrm{V}$,

- declination $\delta \geq 0^{\circ}$.

We isolated 56 MS stars. The list is shown in Table 1. In the close binary $\zeta^{1}$ UMa we could obtain spectra of each of the two companions separately. Thus the total number of target stars is 57 . For those stars that have already been searched for the field by other authors we present the longitudinal (effective) magnetic field $B_{1}$. The most accurate measurements and their references are included in the table. There are 33 stars of luminosity class IV and 5 stars of classes III-IV in the BSC among $V \leq 4$. 0 northern stars. They were not included in the list. Binary stars whose primary (the brighter star) is a MS star were included. The list includes CP stars if they satisfy our criteria. $5 \mathrm{CP}$ stars enter the list in spite of their luminosity class not being indicated in the catalogue.

Our sample represents well MS stars in spectral classes B0.5-F9. The upper spectral type limit B0.5 is defined by the availability of stars brighter than 4 . 0 . The northern stars cooler than F9 are mostly giants, and only 4 of them have luminosity class IV. They do not satisfy our criteria. There are $13 \mathrm{~B}$ stars, $36 \mathrm{~A}$ stars (including $6 \mathrm{Ap}$ and $3 \mathrm{Am}$ ) and $8 \mathrm{~F}$ stars in the list (including one of the coolest $\mathrm{CP}$ star $\beta \mathrm{CrB}$ ). All stars may be considered as belonging to the upper MS stars where one expects global magnetic fields to be present and the convection to be not so strong as to destroy the field. The fraction of Ap stars in the list among stars in the same range of spectral types is $16 \%$. The star $\zeta^{1}$ UMa is probably not an Ap star as reported in BSC. It is classified as having a doubtful nature in The General catalogue of Ap and Am stars (Renson 1991). Our spectra of $\zeta^{1}$ UMa do not show any signs suggesting it is an Ap. Exclusion of $\zeta^{1}$ UMa reduces the fraction of Ap stars to $14 \%$. It is close to the frequencies found by North \& Cramer (1981), Egret \& Jaschek (1981) and Schneider (1994).

The distributions over the sky of the selected stars, stellar magnitude, distance and their projected rotational velocity are shown in Fig. 1. These stars are close to the Sun. Almost all of them are closer than $70 \mathrm{pc}$ (according to the Hipparcos data). In fact, the mixing time for the stars in a volume of $100 \mathrm{pc}$ (assuming their velocity dispersion to be $10-20 \mathrm{~km} \mathrm{~s}^{-1}$ ) is less than their lifetimes. The stars are randomly distributed over the sky. The squeezing of the angular scale to the sky pole causes decreasing of the star density at $\delta>50^{\circ}$. The stars occupy a small volume just inside the galactic plane. Thus a different distance to the cool and hot target stars cannot lead to any selection effects. The projected rotational velocities are in the range $0-350 \mathrm{~km} \mathrm{~s}^{-1}$, and the number of stars monotonically decreases with increasing rotational velocity. The distribution of the sample stars versus rotational velocity is similar to that obtained from greater samples of stars of the same spectral classes (Allen 1977). We can conclude that, with the restrictions mentioned in the beginning of this section, our sample can be considered as unbiased.

Analyzing all published data we find that 4 out of 57 stars of the list have well established magnetic fields ranging between 0.1 and $1.5 \mathrm{kG}$ ( $\epsilon \mathrm{UMa}, \theta$ Aur, $\left.\alpha^{2} \mathrm{CVn}, \beta \mathrm{CrB}\right)$. This suggests that at least $7 \%$ of upper MS stars are magnetic. On the other hand, 24 stars of the list have already been tested for the presence of a field. Therefore, the frequency is less than $17 \%$. It is an upper limit because the stars already studied were the most promising, in expectation of the field. Both the upper and lower limits are rough, but they do not contradict the estimates obtained in Sect. 2. One may expect the real frequency to be closer to the lower limit (7\%), because every star among these 57 has been studied spectroscopically. Probably no other strongly magnetic Ap stars can be found among our targets.

\section{Observations and reduction}

The magnetic survey was carried out on the coude echelle spectrograph CEGS of the $1 \mathrm{~m}$ telescope of the Special Astrophysical Observatory. The instrumental configuration has been described by Musaev (1996). In our observations, incoming light passes through the Zeeman analyzer which contains rotational first quarter-wave plate, a calcite block and second quarter-wave plate. To improve the polarization measurement accuracy the analyzer can operate with two fixed positions of the first quarter-wave plate making an angle of $90^{\circ}$. We used two different orientations of the first quarter-wave plate to record opposite circular polarizations on the same part of a CCD in two adjacent exposures. In this case many instrumental effects can be taken into account (Donati et al. 1990a). 
Table 1. List of stars.

\begin{tabular}{|c|c|c|c|c|c|c|c|}
\hline$\overline{\mathrm{HD}}$ & Name & $\overline{\mathrm{V}}$ & $\overline{\mathrm{Sp}}$ & $\begin{array}{c}v \sin i, \\
\mathrm{~km} \mathrm{~s}^{-1}\end{array}$ & $\begin{array}{r}B_{1}, \\
\mathrm{G}\end{array}$ & $\begin{array}{r}\sigma\left(B_{1}\right), \\
\mathrm{G}\end{array}$ & Reference \\
\hline 4614 & $\eta$ Cas & 3.44 & F9V+dM0 & 6 & 0 & & Gray (1984) \\
\hline 5448 & $\mu$ And & 3.87 & A5V & 72 & & & \\
\hline 11636 & $\beta$ Ari & 2.64 & $\mathrm{~A} 5 \mathrm{~V}$ & 79 & & & \\
\hline 12216 & $50 \mathrm{Cas}$ & 3.98 & $\mathrm{~A} 2 \mathrm{~V}$ & 84 & & & \\
\hline 16970 & $\gamma$ Cet & 3.47 & $\mathrm{~A} 3 \mathrm{~V}$ & 183 & & & \\
\hline 17573 & 41 Ari & 3.63 & $\mathrm{~B} 8 \mathrm{Vn}$ & 180 & & & \\
\hline 19356 & $\beta$ Per & 2.12 & $\mathrm{~B} 8 \mathrm{~V}$ & 65 & 0 & 320 & Borra \& Landstreet (1973) \\
\hline 21364 & $\xi \mathrm{Tau}$ & 3.74 & B9Vn & 33 & & & \\
\hline 24760 & $\epsilon$ Per & 2.89 & $\mathrm{~B} 0.5 \mathrm{~V}+\mathrm{A} 2 \mathrm{~V}$ & 153 & & & \\
\hline 25204 & $\lambda \mathrm{Tau}$ & 3.47 & $\mathrm{~B} 3 \mathrm{~V}+\mathrm{A} 4 \mathrm{IV}$ & 87 & & & \\
\hline 25490 & $v$ Tau & 3.91 & $\mathrm{~A} 1 \mathrm{~V}$ & 69 & & & \\
\hline 30652 & $\pi^{3}$ Ori & 3.19 & F6V & 17 & & & \\
\hline 32630 & $\eta$ Aur & 3.17 & $\mathrm{~B} 3 \mathrm{~V}$ & 132 & & & \\
\hline 40312 & $\theta$ Aur & 2.62 & $\mathrm{~A} 0 \mathrm{pSi}$ & 49 & $-240 \div+360$ & 18 & Borra \& Landstreet (1980) \\
\hline 56537 & $\lambda \mathrm{Gem}$ & 3.58 & $\mathrm{~A} 3 \mathrm{~V}$ & 154 & & & \\
\hline 58715 & $\beta \mathrm{CMi}$ & 2.90 & $\mathrm{~B} 8 \mathrm{Ve}$ & 276 & $-100 \div+240$ & 200 & Barker et al. (1985) \\
\hline 60178 & $\alpha^{2} \mathrm{Gem}$ & 2.88 & $\mathrm{~A} 2 \mathrm{Vm}$ & 30 & 130 & 84 & Landstreet (1982) \\
\hline 60179 & $\alpha^{1} \mathrm{Gem}$ & 1.98 & $\mathrm{~A} 1 \mathrm{~V}$ & 14 & 0 & 37 & Glagolevskij et al. (1989) \\
\hline 76943 & & 3.97 & F5V & 26 & & & \\
\hline 77327 & $\kappa \mathrm{UMa}$ & 3.60 & $\mathrm{~A} 1 \mathrm{Vn}$ & 219 & & & \\
\hline 79469 & $\theta$ Нyа & 3.88 & B9.5V & 81 & & & \\
\hline 80081 & 38 Lyn & 3.82 & $\mathrm{~A} 3 \mathrm{~V}$ & 165 & & & \\
\hline 87901 & $\alpha$ Leo & 1.35 & B7V & 329 & -15 & 65 & Landstreet (1982) \\
\hline 95418 & $\beta \mathrm{UMa}$ & 2.37 & A1V & 39 & 0 & 65 & Landstreet (1982) \\
\hline 97603 & $\delta$ Leo & 2.56 & $\mathrm{~A} 4 \mathrm{~V}$ & 181 & -75 & 65 & Landstreet (1982) \\
\hline 97633 & $\theta$ Leo & 3.34 & $\mathrm{~A} 2 \mathrm{~V}$ & 20 & -49 & 55 & Landstreet (1982) \\
\hline 102647 & $\beta$ Leo & 2.14 & $\mathrm{~A} 3 \mathrm{~V}$ & 121 & -80 & 65 & Landstreet (1982) \\
\hline 102870 & $\beta$ Vir & 3.61 & F9V & 3 & 0 & & Gray (1984) \\
\hline 103287 & $\gamma \mathrm{UMa}$ & 2.44 & $\mathrm{~A} 0 \mathrm{Ve}$ & 168 & 25 & 50 & Landstreet (1982) \\
\hline 106591 & $\delta \mathrm{UMa}$ & 3.31 & $\mathrm{~A} 3 \mathrm{~V}$ & 177 & & & \\
\hline 112185 & $\epsilon \mathrm{UMa}$ & 1.77 & $\mathrm{~A} 0 \mathrm{pCr}$ & 38 & $-30 \div+90$ & 18 & Wade et al. (2000) \\
\hline 112413 & $\alpha^{2} \mathrm{CVn}$ & 2.90 & $\mathrm{~A} 0 \mathrm{pSiEuHg}$ & 29 & $-730 \div+630$ & 55 & Wade et al. (2000) \\
\hline 116656 & $\zeta^{1} \mathrm{UMa}$ & 2.27 & $\mathrm{~A} 1 \mathrm{VpSrSi}$ & 32 & & & \\
\hline 116657 & $\zeta^{2} \mathrm{UMa}$ & 3.95 & $\mathrm{~A} 1 \mathrm{~m}$ & 57 & & & \\
\hline 120315 & $\eta \mathrm{UMa}$ & 1.86 & $\mathrm{~B} 3 \mathrm{~V}$ & 205 & -5 & 65 & Landstreet (1982) \\
\hline 130109 & 109 Vir & 3.72 & $\mathrm{~A} 0 \mathrm{~V}$ & 351 & & & \\
\hline 137909 & $\beta \mathrm{CrB}$ & 3.68 & F0p & 19 & $-570 \div+760$ & 21 & Wade et al. (2000) \\
\hline 139006 & $\alpha \mathrm{CrB}$ & 2.23 & $\mathrm{~A} 0 \mathrm{~V}+\mathrm{G} 5 \mathrm{~V}$ & 133 & & & \\
\hline 141795 & $\epsilon$ Ser & 3.71 & $\mathrm{~A} 2 \mathrm{Vm}$ & 37 & -60 & 48 & Landstreet (1982) \\
\hline 142860 & $\gamma$ Ser & 3.85 & F6V & 8 & -142 & 161 & Boesgaard (1974) \\
\hline 148857 & $\lambda \mathrm{Oph}$ & 3.82 & $\mathrm{~A} 0 \mathrm{~V}+\mathrm{A} 4 \mathrm{~V}$ & 127 & & & \\
\hline 153808 & $\epsilon$ Her & 3.92 & $\mathrm{~A} 0 \mathrm{~V}$ & 78 & & & \\
\hline 161868 & $\gamma \mathrm{Oph}$ & 3.75 & A0Vnp & 205 & & & \\
\hline 166014 & $o$ Her & 3.83 & B9.5V & 134 & & & \\
\hline 170153 & $\chi$ Dra & 3.57 & F7V & 11 & 0 & & Gray (1984) \\
\hline 172167 & $\alpha$ Lyr & 0.03 & A0Va & 15 & -9 & 19 & Landstreet (1982) \\
\hline 177724 & $\zeta \mathrm{Aql}$ & 2.99 & A0Vn & 331 & & & \\
\hline 184006 & $\iota^{2} \mathrm{Cyg}$ & 3.79 & $\mathrm{~A} 5 \mathrm{Vn}$ & 226 & & & \\
\hline 187642 & $\alpha \mathrm{Aql}$ & 0.77 & A7V & 242 & -65 & 60 & Landstreet (1982) \\
\hline 199629 & $v$ Cyg & 3.94 & $\mathrm{~A} 1 \mathrm{Vn}$ & 241 & & & \\
\hline 203280 & $\alpha$ Cep & 2.44 & A7V & 246 & & & \\
\hline 210027 & $\iota \mathrm{Peg}$ & 3.76 & F5V & 7 & -13 & 31 & Boesgaard (1974) \\
\hline 210418 & $\theta$ Peg & 3.53 & $\mathrm{~A} 2 \mathrm{Vp}$ & 117 & $-80 \div+30$ & 170 & Bohlender \& Landstreet (1990a) \\
\hline 213558 & $\alpha \mathrm{Lac}$ & 3.77 & $\mathrm{~A} 1 \mathrm{~V}$ & 146 & & & \\
\hline 214923 & $\zeta$ Peg & 3.40 & $\mathrm{~B} 8 \mathrm{~V}$ & 194 & & & \\
\hline 218045 & $\alpha$ Peg & 2.49 & B9V & 148 & & & \\
\hline
\end{tabular}



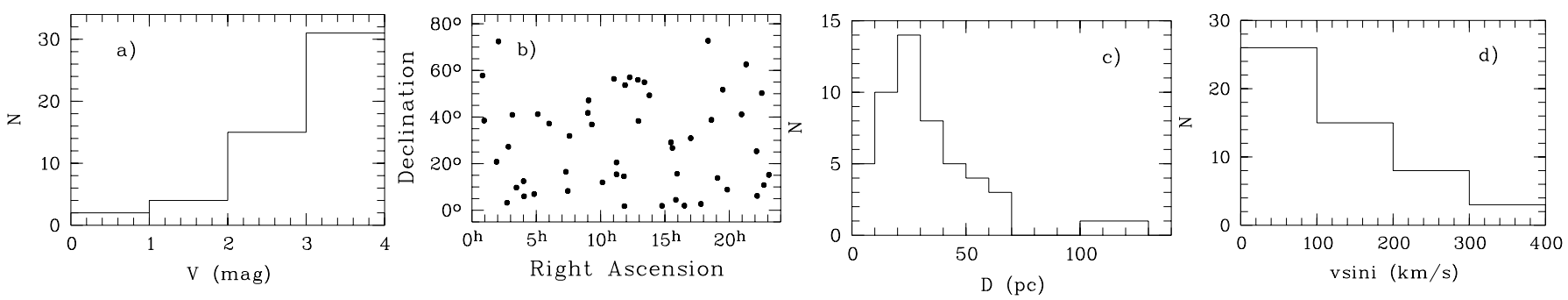

Fig. 1. Distributions of the target stars versus a) stellar magnitude, b) over the sky, c) versus distance and d) rotational velocity.

Left- and right-circularly polarized (LCP and RCP) Zeeman spectra are obtained simultaneously on the Wright Instruments $\mathrm{CCD}(1242 \times 1152$ pixels $)$. With the entrance slit set to $2^{\prime \prime}$ the instrument configuration yields a resolving power of about $R=40000$ over a spectral range from 3700 to $9000 \AA$.

The spectra were taken between March 1996 and June 2000. The total number of stars observed in this period is 30. Exposure times were chosen to be longer for fainter stars so that the $S / N$ ratio in their spectra was nearly the same as in the spectra of brighter stars.

The data reduction was carried out using the image processing package MIDAS with some modifications for polarimetric spectra (Monin 1999). The general steps are as follows: definition of spectral orders, fitting and removal of the background scattered light, cosmic ray hit removal, extraction of the spectral orders in opposite polarizations, normalization to the continuum, determination of a dispersion law, line identification, magnetic shift determination. The background is approximated by a smoothing spline interpolation between interorder points. The extraction of orders is carried out using the optimal extraction algorithm based on that described by Mukai (1990).

The transmission of LCP and RCP components through the spectrograph are slightly different. Therefore, a spurious broadband polarization signal arises. Its value changes both along the spectral order and from one order to another. As was mentioned above, to reduce most of the instrumental effects we make two exposures with different orientations of the first quarter-wave plate. Nevertheless, a small difference between LCP and RCP fluxes averaged in two exposures may exist if the exposures are obtained under slightly different conditions. Thus we need to normalize spectra or define well the continuum shape. Precise magnetic shift measurements require an accurate continuum shape reconstruction. The continuum fitting is made using an algorithm based on a smoothing-andclipping high-frequency filter (Shergin et al. 1996). We use a convolution with a Gaussian profile for the smoothing. The algorithm takes the noise level into account.

Broad absorption lines make it difficult to determine the underlying continuum. The full width of hydrogen lines may reach tens of Angströms and line wings may spread far beyond the spectral order. The line wings can appear even in adjacent orders. To restore the true continuum flux in the region containing a broad line, we interpolate the continuum flux in several adjacent orders. A low degree (2nd or 3rd order) polynomial interpolation is applied. We use the same fitting parameters for both polarizations. It is difficult to reconstruct the continuum flux near the Balmer and Paschen jumps. Beyond the Balmer jump the spectra' quality is not sufficient for our goals. In the red part (longer than $7000 \AA$ ) almost all orders contain strong telluric bands. These orders cannot be used in the reconstruction procedure. In all other spectral regions the algorithm works well even in the case of a complicated instrumental response function.

A spectrum of scattered solar light is used as a reference spectrum for wavelength calibration. The spectrum is corrected for both Earth's rotation and orbital motion. To reach high accuracy in the determination of the dispersion law we identify at least 20 lines uniformly distributed over the image. We use the line list from Pierce \& Breckinridge (1973). The dispersion relation can be expressed as: $m \lambda=f(x, 1 / m)$, where $m$ is the absolute order number and $x$ is the pixel position along the direction of the main dispersion. $f(x, 1 / m)$ is a polynomial function. The standard deviation in individual line positions was typically about $0.003 \AA$. In the worst cases it did not exceed $0.007 \AA$.

For spectral line identification we use the line lists formed by the database VALD (Piskunov et al. 1995; Kupka et al. 1999; Ryabchikova at al. 1999). This database gives also the latest effective Lande factors of lines of many chemical elements (including rare-earth ones).

Magnetic shifts are measured using two different methods: the center-of-gravity, the classical method developed for photographic technique, and the Fourier cross-correlation method. In the first case, we obtain the displacement of the center of gravity of spectral lines in opposite circular polarizations:

$\Delta \lambda=\lambda_{\mathrm{R}}-\lambda_{\mathrm{L}}=9.34 \times 10^{-13} g_{\text {eff }} B_{1} \lambda^{2}$,

where $g_{\text {eff }}$ - effective Lande factor, $B_{1}$ - longitudinal magnetic field and $\lambda$-wavelength. In the second case, we can evaluate the displacement of line profiles as a whole. Application of the last method to magnetic shift measurements has been tested by Klochkova et al. (1996). It is less sensitive to overestimation or underestimation of the continuum level, so this method can be applied in those parts of spectra where it is difficult to normalize to the continuum adequately.

In fact, the accuracy of magnetic shift measurements depends on many parameters. The most important are (in order of importance) the number of lines measured, the spectra quality (signal-to-noise ratio $S / N$ ), the line intensity and width $(v \sin i)$, and the method used for magnetic shift measurement. Less important are the line shape, blending, accuracy of Lande factor of individual lines, instrumental polarization and depolarization. 
Taking into account the accuracy of individual line measurements improves considerably the resulting accuracy. Donati et al. (1997) showed that extremally small Zeeman signatures could be detected from the analysis of many spectral lines. They suggested analysis of a mean Stokes $V$ profile, reproduced by the Stokes $V$ profiles of all selected lines. The optimal weighting was $g_{\mathrm{eff}} \cdot \lambda \cdot r_{\mathrm{c}} \cdot(S / N)^{2}$, where $r_{\mathrm{c}}$ is central depth of the line. Instead of averaging individual Stokes $V$ profiles, we calculated the magnetic shift for each individual line using Eq. (1). The measurements of individual lines were averaged using $r_{\mathrm{c}}$ or $S / N$ as a weight $w$ :

$$
B_{1}=\frac{\sum_{i=1}^{n} B_{1}^{(i)} w_{i}}{\sum_{i=1}^{n} w_{i}}
$$

the accuracy of $B_{1}$ is:

$$
\sigma\left(B_{1}\right)=\frac{\sqrt{\sum_{i=1}^{n}\left(B_{1}^{(i)}-B_{1}\right)^{2} w_{i}^{2}}}{\sum_{i=1}^{n} w_{i}} .
$$

The accuracy of individual line measurements depends mainly on $r_{\mathrm{c}}$ and $S / N . B_{1}$ given by Eq. (1) contains $g_{\mathrm{eff}}$ and $\lambda$, and it is not necessary to include them in weighting. Valyavin et al. (1997) showed that for good quality spectra $(S / N>70)$ the line intensity $r_{\mathrm{c}}$ is the first factor influencing uncertainties in the magnetic shift measurements. In our spectra $S / N$ do not significantly vary. Taking the variations of $S / N$ into account does not considerably improve the accuracy. Instead, $r_{\mathrm{c}}$ of individual lines varies over a wide range, 0.03-0.90. As a result, the accuracy of the measurements varies significantly from line to line. Thus, variations of $r_{\mathrm{c}}$ primarily should be taken into account, $w=r_{\mathrm{c}}$.

The central depth of hydrogen lines does not vary significantly from line to line. Thus, it is better to weight magnetic shifts of hydrogen lines by the $S / N$ ratio, $w=S / N$. H $\alpha$ has a maximum weight. The throughput of the spectrograph and detector decreases toward blue orders. Hydrogen lines which are close to the Balmer jump give a smaller contribution to the weighted average $B_{1}$ value. Pashen lines also have lower weight because of spectra quality degradation in orders with wavelengths longer than about $7000 \AA$.

Using weighted values we improve the precision of magnetic field estimates. In some cases, for example, when the number of measurable spectral lines is small, it allows us to reduce the uncertainty by a factor of 2 .

In spectra of rapidly rotating stars the magnetic shifts are measured in a central part of the line profile (about $\pm 10 \AA$ ). Line wings are less sensitive to the magnetic shift because of a reducing of the profile gradient. Therefore, taking a broader band around the line center, we do not improve the accuracy. In broad lines even small errors in the continuum restoration may introduce a spurious polarization signal. This signal becomes sufficient when we analyze a considerable part of the line profile. On the other hand, we get worse accuracies if we take the band too narrowly. The line center is essentially flattened by fast rotation. We have found an optimal range, $\pm 5 \div \pm 10 \AA$, which provides the best accuracy.

We checked our measurements for spurious polarization using telluric lines. When the number of identified telluric lines is 100-300 we can reach an extremely high precision of about a few $\mathrm{G}$. We concluded that no spurious polarization signals due to the influence of uncorrected instrumental effects are present.

We have observed two well-known magnetic stars, 53 Cam and $\epsilon \mathrm{UMa}$. $53 \mathrm{Cam}$ is an Ap star whose longitudinal magnetic field varies between -5 and $+5 \mathrm{kG}$, the rotational period is 8.0268 (Hill 1998). It is fainter $(V=6.01)$ than our targets. Nevertheless, we have obtained good quality spectra in the region 5000-6500 $\AA$ in different phases of the rotational period. Two $B_{1}$ estimates were made near the positive extreme while two others were obtained when the longitudinal magnetic field reversed its sign and became negative. About 30 unblended lines with a particularly simple Zeeman pattern were selected. Most of them are Fe I lines (70\%). All measurements were phased according to the ephemeris of Hill et al. (1998). Our measurements agree well with published data (see Fig. 2).

One of the goals of our survey is to search for magnetic fields with extremely high accuracy. In Fig. 3 we present the comparison of our measurements of the weakfield star $\epsilon$ UMa with those obtained by other investigators: the Balmer-line Zeeman analyzer measurements reported by Bohlender \& Landstreet (1990b), and LSD $B_{1}$ measurements by Wade et al. (2000). $\epsilon$ UMa has the weakest global magnetic field, its strength varies between approximately -100 and $+100 \mathrm{G}$ while the star rotates. Most of the time the longitudinal magnetic field of $\epsilon \mathrm{UMa}$ is below the limit of detectability which can be reached by the polarimetric instruments made so far. The measurements were phased according to the ephemeris of Provin (1953). Our $B_{1}$ estimates, $+93 \pm 26$ and $+107 \pm 33 \mathrm{G}$, agree well with the previous determinations (see Fig. 3), and a very good internal consistency between the two independent measurements exists. We note that internal consistency of individual $B_{1}$ measurements in those stars which have been repeatedly observed is always less than an error estimated using Eq. (3). Therefore, we suppose that the accuracy of our magnetic field measurements may be even better than we indicate. The magnetic shift in the $\mathrm{H} \alpha$ core of $\epsilon \mathrm{UMa}$ corresponds to a slightly lower magnetic field, +89 and $+80 \mathrm{G}$. Such a difference may be explained in terms of the concentration of $\mathrm{Fe}$ and $\mathrm{Cr}$ in the magnetic poles (Rice \& Wehlay 1991). Our $B_{1}$ measurements were obtained near a phase when the positive magnetic pole was seen.

We can conclude that our data reduction software behaves properly. Our $B_{1}$ measurements of known magnetic stars are in agreement with those obtained by other authors. We are able to detect both strong and very weak stellar magnetic fields.

\section{Results}

We have measured longitudinal magnetic fields in 30 of the 57 isolated stars. Results of the measurements are presented in Table 2. The columns give in consecutive order the name or HD number, heliocentric Julian date of the middle of an observation, the longitudinal magnetic field $B_{1}$ with corresponding 


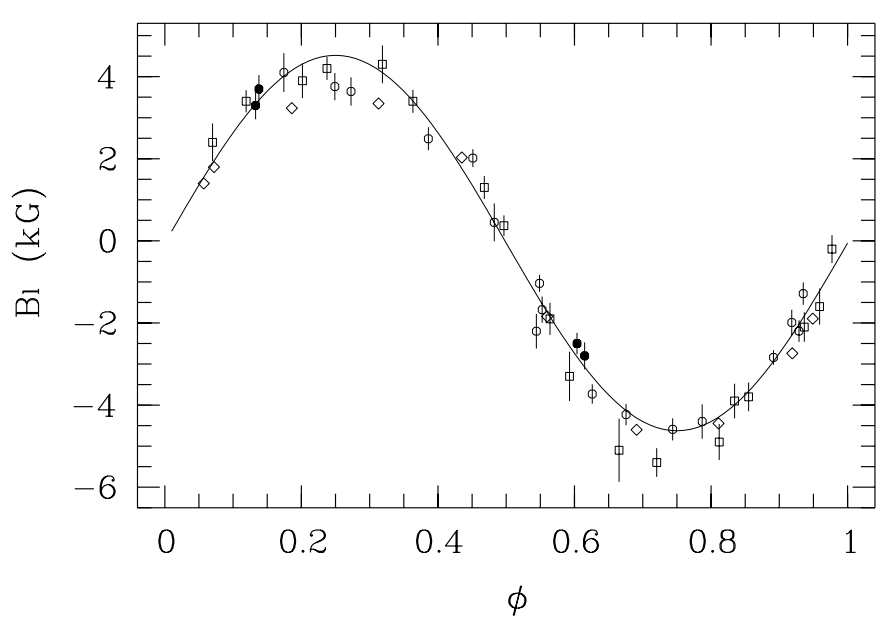

Fig. 2. Longitudinal magnetic field of $53 \mathrm{Cam}$ against rotational phase. Open circles - the data from Hill et al. (1998). Open square - the data from Borra \& Landstreet (1977). Open diamonds - the data by Wade et al. (2000) obtained from LSD profiles. Filled circles - our measurements. The fitting curve by Hill et al. (1998) is presented. The error bars that correspond to one standard deviation are shown.

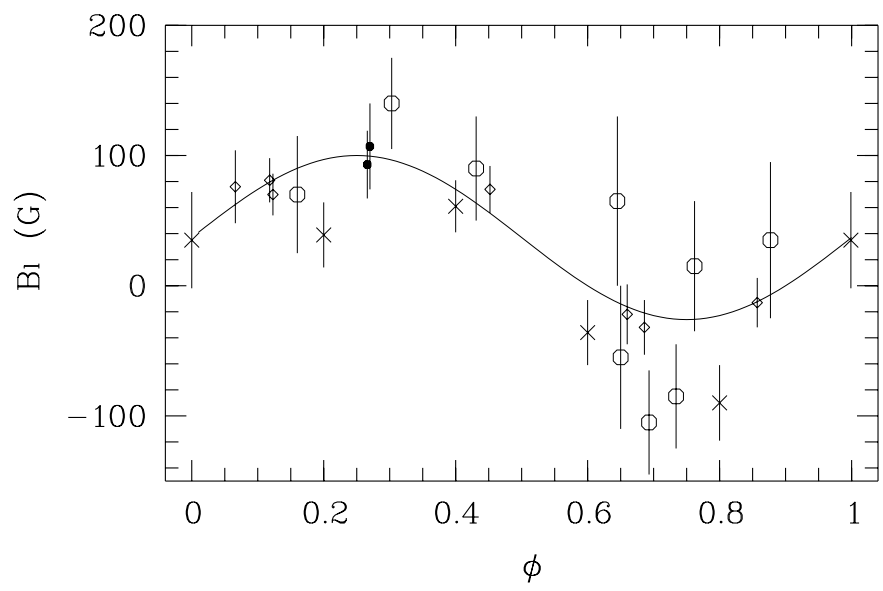

Fig. 3. Longitudinal magnetic field of $\epsilon$ UMa against rotational phase. Open circles - the data from Bohlender \& Landstreet (1990b). Crosses - the data from Donati et al. (1990b). Open diamonds are $B_{1}$ measurements obtained from LSD profiles by Wade et al. (2000). Filled circles - our measurements. The error bars that correspond to one standard deviation are shown.

errors evaluated according to Eq. (3), and the number $n$ of spectral lines used. For some stars the results for hydrogen lines are presented separately. Both the center-of-gravity $\left(B_{1}^{\mathrm{cg}}\right)$ and the Fourier cross-correlation $\left(B_{1}^{\mathrm{fcc}}\right)$ measurements are shown. Each $B_{1}$ value was obtained using two exposures with a different orientation of the first quarter-wave plate of the analyzer.

The accuracy varies from star to star. The main factor affecting the precision is the number of lines measured $n$. In Fig. 4 we show the measurement uncertainties against $n$, plotted from the data in Table 2. When the number of lines is less than 10 or more than 200 the accuracy is roughly independent of $n$. It appears that the smallest error which can be reached is $10-15$ G. It is almost independent of the brightness of a star, because we have taken spectra of about the same quality for both bright and faint stars. A residual instrumental effect that

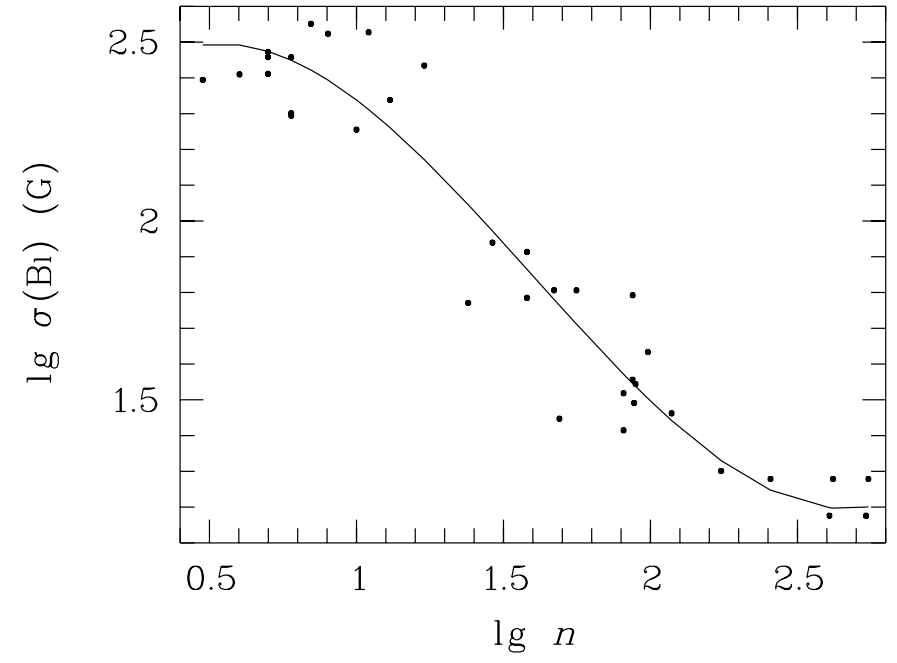

Fig. 4. The measurement uncertainties of $B_{1}$ against the number of measured lines. The solid line is the best fit of the points.

cannot be removed even by the differential measurements lead to the precision limitation at the level of $10 \mathrm{G}$. When the number of measurable lines is small $n<10$ (most of the lines have low contrast and only hydrogen lines can be analyzed), the precision tends to be around 200-300 G. Spectra' quality (the $S / N$ ratio) drops rapidly from $\mathrm{H} \alpha$ to higher Balmer lines. Thus $\mathrm{H} \alpha$ has a weight which is considerably greater than those of other hydrogen lines, and inclusion of other hydrogen lines does not change the result significantly. This explains the behavior of the errors in Fig. 4 at small $n$.

For photon noise limited measurements, the standard error is given by $\sigma\left(B_{1}\right) \propto n^{-0.5}$. From Fig. 4 we find $\sigma\left(B_{1}\right) \propto n^{-0.8 \pm 0.1}$ when $n$ ranges from 10 to 100 . The observed slope is steeper than expected. It is caused by the fact that contribution of cool stars increases when $n$ grows. The cool stars, whose spectra are rich in lines, are usually slow rotators, and they have higher line contrast. The field measurement error depends on the line contrast. This results in a higher accuracy than expected from the square root dependency.

In the majority of stars in our list we did not detect magnetic fields. However in some stars one may suspect a positive detection. They are the star $\theta$ Leo with distinct features of chemical anomalies of the Am type (Savanov 1985), the star $v$ Cyg showing the presence of variable Be-like emission in $\mathrm{H} \alpha$, the fast rotating star $\alpha \mathrm{Aql}$, and one of the coolest star in the list, $\chi$ Dra. We intend to discuss the most interesting stars in our next paper. Here we discuss in detail two stars, $\beta$ Vir and $\chi$ Dra. These stars are very similar in temperature, brightness and $v \sin i$, but while the first star shows a null result, there is evidence of a weak field in the second one.

\section{$\beta \operatorname{Vir}(H D$ 102870, HR 4540)}

The first attempt to detect a magnetic field in this star was made by Gray (1984) by searching for Zeeman broadening in spectral lines. He detected no magnetic field. Andreetta \& Giampapa (1995) concluded that only $7 \%$ of the surface 
Table 2. The magnetic field measurements.

\begin{tabular}{|c|c|c|c|c|c|c|c|c|c|}
\hline \multirow[t]{2}{*}{ Name } & \multirow{2}{*}{$\begin{array}{r}\text { JD + } \\
2450000\end{array}$} & \multicolumn{4}{|c|}{ All lines } & \multicolumn{4}{|c|}{ Hydrogen lines } \\
\hline & & $B_{1}^{\mathrm{cg}}, \mathrm{G}$ & $n$ & $B_{1}^{\mathrm{fcc}}, \mathrm{G}$ & $n$ & $B_{1}^{\mathrm{cg}}, \mathrm{G}$ & $n$ & $B_{1}^{\mathrm{fcc}}, \mathrm{G}$ & $n$ \\
\hline$\gamma \mathrm{Cet}$ & 327.87 & & & & & $-70 \pm 300$ & 5 & $-220 \pm 390$ & 5 \\
\hline$\alpha^{2} \mathrm{Gem}$ & 1659.30 & $-41 \pm 29$ & 118 & $-22 \pm 32$ & 123 & & & & \\
\hline$\alpha^{1} \mathrm{Gem}$ & 1659.33 & $9 \pm 43$ & 98 & $13 \pm 30$ & 97 & & & & \\
\hline HD76943 & 1658.27 & $13 \pm 20$ & 174 & $-6 \pm 23$ & 176 & & & & \\
\hline$\kappa \mathrm{UMa}$ & 1658.34 & $-470 \pm 330$ & 8 & $-660 \pm 360$ & 8 & $-640 \pm 370$ & 7 & $-570 \pm 400$ & 7 \\
\hline$\theta$ Нyа & 1661.27 & $140 \pm 95$ & 7 & $-30 \pm 130$ & 8 & $200 \pm 150$ & 4 & $59 \pm 91$ & 4 \\
\hline 38 Lyn & 1660.32 & $-410 \pm 290$ & 5 & $-600 \pm 300$ & 5 & $-270 \pm 350$ & 4 & $-420 \pm 380$ & 4 \\
\hline$\beta \mathrm{UMa}$ & 177.25 & $72 \pm 64$ & 47 & $7 \pm 90$ & 46 & & & & \\
\hline \multirow[t]{3}{*}{$\theta$ Leo } & 1595.41 & $19 \pm 31$ & 88 & $24 \pm 34$ & 93 & 55 & 1 & & \\
\hline & 1595.46 & $73 \pm 36$ & 87 & $58 \pm 35$ & 94 & 31 & 1 & & \\
\hline & 1595.53 & $64 \pm 35$ & 89 & $38 \pm 30$ & 93 & 82 & 1 & & \\
\hline$\beta$ Vir & 589.27 & $-10 \pm 19$ & 418 & $-3 \pm 18$ & 423 & & & & \\
\hline$\gamma \mathrm{UMa}$ & 177.29 & $-280 \pm 270$ & 17 & $-360 \pm 280$ & 16 & $-290 \pm 270$ & 13 & $-140 \pm 230$ & 13 \\
\hline$\delta \mathrm{UMa}$ & 177.33 & $50 \pm 220$ & 13 & $-450 \pm 380$ & 13 & $-50 \pm 300$ & 10 & $-470 \pm 230$ & 10 \\
\hline \multirow[t]{2}{*}{$\epsilon \mathrm{UMa}$} & 1661.31 & $93 \pm 26$ & 81 & $95 \pm 30$ & 102 & 89 & 1 & 85 & 1 \\
\hline & 1661.34 & $107 \pm 33$ & 81 & $107 \pm 36$ & 97 & 80 & 1 & 81 & 1 \\
\hline \multirow[t]{2}{*}{$\zeta^{1} \mathrm{UMa} \mathrm{A}$} & 177.38 & $-91 \pm 82$ & 38 & $-170 \pm 110$ & 38 & & & & \\
\hline & 177.44 & $26 \pm 61$ & 38 & $49 \pm 63$ & 36 & & & & \\
\hline \multirow[t]{2}{*}{$\zeta^{1} \mathrm{UMaB}$} & 177.38 & $33 \pm 87$ & 29 & $50 \pm 140$ & 27 & & & & \\
\hline & 177.44 & $-6 \pm 59$ & 24 & $-95 \pm 88$ & 24 & & & & \\
\hline$\zeta^{2} \mathrm{UMa}$ & 1657.34 & $19 \pm 64$ & 56 & $58 \pm 49$ & 57 & & & & \\
\hline$\epsilon$ Ser & 588.36 & $21 \pm 62$ & 87 & $20 \pm 67$ & 118 & & & & \\
\hline$\gamma \mathrm{Ser}$ & 327.61 & $-5 \pm 19$ & 256 & $-6 \pm 17$ & 269 & & & & \\
\hline$\gamma \mathrm{Oph}$ & 588.48 & & & & & $-10 \pm 260$ & 4 & $350 \pm 320$ & 4 \\
\hline$o$ Her & 1658.47 & & & & & $400 \pm 200$ & 6 & $140 \pm 210$ & 6 \\
\hline \multirow[t]{2}{*}{$\chi$ Dra } & 326.58 & $-42 \pm 19$ & 551 & $-21 \pm 10$ & 549 & -67 & 1 & -72 & 1 \\
\hline & 326.60 & $-47 \pm 15$ & 541 & $-25 \pm 9$ & 539 & -61 & 1 & -60 & 1 \\
\hline$\alpha$ Lyr & 1362.43 & $3 \pm 28$ & 49 & $-20 \pm 20$ & 61 & & & & \\
\hline$\zeta \mathrm{Aql}$ & 326.71 & & & & & $-190 \pm 290$ & 6 & $-420 \pm 340$ & 6 \\
\hline$\alpha \mathrm{Aql}$ & 1363.44 & $100 \pm 180$ & 10 & $173 \pm 78$ & 11 & $122 \pm 35$ & 4 & $115 \pm 42$ & 4 \\
\hline \multirow[t]{2}{*}{$v$ Cyg } & 326.84 & & & & & $140 \pm 360$ & 7 & $-260 \pm 270$ & 7 \\
\hline & 1362.50 & & & & & $630 \pm 250$ & 3 & $700 \pm 240$ & 3 \\
\hline$\alpha$ Cep & 589.52 & & & & & $-30 \pm 89$ & 4 & $60 \pm 310$ & 8 \\
\hline$\iota \mathrm{Peg}$ & 327.71 & $6 \pm 15$ & 406 & $6 \pm 10$ & 415 & & & & \\
\hline$\theta$ Peg & 327.77 & $200 \pm 340$ & 11 & $-370 \pm 300$ & 15 & $280 \pm 350$ & 6 & $-40 \pm 430$ & 6 \\
\hline$\alpha \mathrm{Lac}$ & 1363.49 & $-200 \pm 200$ & 6 & $-110 \pm 240$ & 7 & & & & \\
\hline$\zeta$ Peg & 327.82 & & & & & $-30 \pm 260$ & 5 & $-40 \pm 260$ & 5 \\
\hline
\end{tabular}

of $\beta$ Vir may be covered by magnetic regions. They estimated the fractional area covered by active, magnetic regions.

We used more than 400 lines for the measurements. Equivalent width of the identified lines ranges from 20 to $300 \mathrm{~m} \AA$. The list of chemical elements having the most numerous lines is presented in Table 3. It contains the number of lines and magnetic shifts derived from lines of the given species. Apart from those elements we used also spectral lines of Ti, Mn, Sc, Co, Cr. The magnetic field obtained from all the lines is null with an accuracy of $\sigma=19 \mathrm{G}$ (see Table 2).
Both the center-of-gravity and the Fourier cross-correlation methods give the same result.

A large number of lines in the spectra of $\beta$ Vir allowed us to check possible systematic errors of our magnetic field estimates. We studied the magnetic shifts versus various spectral line parameters such as Lande factor, wavelength, equivalent width, and equivalent width error. In all the cases we did not find any correlation, the correlation coefficients being less than 0.05 . We conclude that there are no notable systematic errors in our data. 
Table 3. Elements with the most numerous lines and magnetic field in $\beta$ Vir.

\begin{tabular}{crr}
\hline \hline Element & $n$ & \multicolumn{1}{c}{$B_{1}^{\text {cg }}, \mathrm{G}$} \\
\hline Fe I & 259 & $8 \pm 21$ \\
Fe II & 20 & $21 \pm 66$ \\
Ni I & 47 & $-85 \pm 72$ \\
Ca I & 22 & $-54 \pm 98$ \\
\hline
\end{tabular}

\section{$\chi \operatorname{Dra}(H D$ 170153, HR 6927)}

$\chi$ Dra is a spectroscopic binary. Its orbital period is 280.55 days. The angular separation between components is 0.'12 (Tomkin et al. 1987; Schoeller et al. 1998). Tomkin et al. (1987) reported elements of both spectroscopic and interferometric orbits. At the time of our observations spectral lines of the primary and secondary components were unresolved (the orbital phase $\approx 0.4$ ). The radial velocity in our composite spectra $32.8 \pm 0.1 \mathrm{~km} \mathrm{~s}^{-1}$ is close to the systemic velocity $32.5 \pm 0.1 \mathrm{~km} \mathrm{~s}^{-1}$. Because of the high orbital inclination $i=75^{\circ} \pm 1^{\circ}$ eclipses in the binary do not occur. This means that spectra near the phase $\approx 0.4$ consist of lines of both components and the positions of lines of both stars coincide. Spectral classes of the stars were determined by Tomkin et al. (1987) as F7V for the primary and G8V (or K2V) for the secondary.

Gray (1984) tried to detect the field in $\chi$ Dra searching for Zeeman broadening in 16 spectral lines. The magnetic field was not detected in that study. We cannot compare our data with the results by Gray (1984) because he did not publish an accuracy.

We have identified 124 telluric lines between 5800 and $9000 \AA$ A. The central depth ranges from 0.05 to 0.9 . We used these lines to check the magnetic field measurements. Telluric lines are very narrow with steep profile gradients. No shifts in the telluric lines were found. Accepting the Lande factor equal to 1 we found the formal magnetic shift in telluric lines $18 \pm 14 \mathrm{G}$ and $-10 \pm 15 \mathrm{G}$. This fact along with others allows us to exclude any systematic instrumental effects.

The spectrum has numerous sharp lines $(v \sin i=$ $11 \mathrm{~km} \mathrm{~s}^{-1}$ ). We identified more than 500 lines in spectral region $4000-8700 \AA$. Most of them are Fe lines (59\%). Fe I lines are much more numerous than Fe II lines (4\%). In Table 4 we present results of measurements by the center-of-gravity method of those elements that have significant number of lines in the spectra of $\chi$ Dra. It corresponds to the second measurement according to Table 2, JD 2450326.60.

$\mathrm{Ti}$ (9\% of all identified lines), Ni (8\%), Cr (5\%), Ca (3\%) and $\mathrm{Mn}(3 \%)$ lines do not show reliable magnetic shift. Fe I lines give the main contribution to the magnetic field estimates, $B_{1}=-32 \pm 20$ and $B_{1}=-55 \pm 17$. Using Fe II lines we found a higher field strength, $-94 \pm 53 \mathrm{G}$ and $-200 \pm 42$. The difference between two average values $-44 \mathrm{G}$ for the Fe I lines and $-147 \mathrm{G}$ for the Fe II lines is about 3 error bars. This difference could be, in principle, a statistical effect. On the other hand, the primary and secondary lines in $\chi$ Dra were unresolved in our observations. The secondary has an effective temperature of $4900 \mathrm{~K}$ which is less than temperature of the primary by $1200 \mathrm{~K}$ (Tomkin et al. 1987). Using speckle interferometric
Table 4. The magnetic field of $\chi$ Dra derived from chemical elements that have a significant number of lines.

\begin{tabular}{lrr}
\hline \hline Element & $n$ & $B_{1}^{\mathrm{cg}}, \mathrm{G}$ \\
\hline $\mathrm{Fe} \mathrm{I}$ & 326 & $-55 \pm 17$ \\
$\mathrm{Fe} \mathrm{II}$ & 20 & $-200 \pm 42$ \\
$\mathrm{Ti}$ & 50 & $14 \pm 48$ \\
$\mathrm{Ni}$ & 45 & $-4 \pm 51$ \\
$\mathrm{Cr}$ & 28 & $47 \pm 82$ \\
$\mathrm{Ca}$ & 19 & $-26 \pm 68$ \\
$\mathrm{Mn}$ & 16 & $-6 \pm 75$ \\
\hline
\end{tabular}

data Schoeller et al. (1998) reported that the stellar magnitude of the components differs by about 2 in the red (6500-7500 $\AA$ ). Some spectral lines are formed almost completely in the hotter star, but the cooler component becomes noticeable in others. The Fe II lines are formed mainly in the atmosphere of primary. Therefore, the difference in the field strength mentioned above may also be due to depolarization by the lines of the secondary star.

To check this hypothesis we found a line ratio $\gamma=r_{\mathrm{sec}} / r_{\mathrm{pri}}$ (secondary over primary) in the composite spectrum. We used line center intensities from two models, $T_{\text {eff }}=5000 \mathrm{~K}$ and $T_{\text {eff }}=6000 \mathrm{~K}$. The models were taken from the VALD database. We found the line ratio for each line identified in the spectrum. The flux difference of two components (by about a factor of 6 in the red and 9 in the blue part of the spectra) was taken into account. When $\gamma$ is small it means that the line is formed mainly in the hotter star. When $\gamma=1$, lines are equally due to primary and secondary. If the primary star is magnetic and secondary is non-magnetic or it has a longitudinal field of opposite polarity, we may expect a decrease of the field strength derived from individual lines with increasing of $\gamma$. It may even change its sign at some $\gamma$ if the field of the secondary is strong enough. In Fig. 5 the longitudinal magnetic field of individual lines derived from the second pair of spectra (JD 2450 326.60) is plotted against $\gamma$. The Fe II lines are strongly concentrated in the region where $\gamma<0.1$. The Fe I lines are almost uniformly distributed in the range $0.1<\gamma<0.25$. The behavior of mean values of the field does not contradict the hypothesis of light depolarization by the secondary, but the precision of the mean values outside the region $0.1<\gamma<0.15$ is low. We cannot distinguish now whether the difference between Fe I and Fe II is due to the depolarization or if it is a statistical effect only.

We also apply another method to detect the field in the spectra of $\chi$ Dra. Spectral line magnetic splitting $(\Delta \lambda)$ depends on the Lande factor $\left(g_{\text {eff }}\right)$ and the line wavelength. We can introduce a reduced magnetic shift

$A=B_{1} \cdot g_{\text {eff }}=1.07 \times 10^{12} \Delta \lambda / \lambda^{2}$,

where wavelength is given in Angströms. The parameter $A$ is proportional to $g_{\text {eff }}$ with the longitudinal magnetic field as a constant term. The reduced magnetic shift as a function of $g_{\text {eff }}$ is presented in Fig. 6. We used 494 lines whose Lande factors range from 0.8 to 2.6. All the lines were binned in 4 intervals of $g_{\mathrm{eff}}$ : 0.8-1.2 (215 lines), 1.2-1.6 (207 lines), 


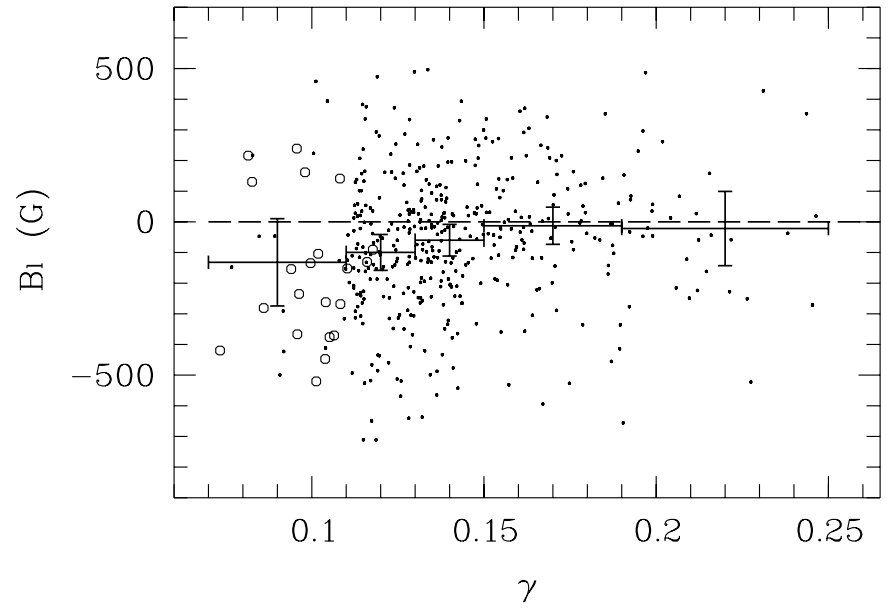

Fig. 5. The longitudinal magnetic field of $\chi$ Dra against the secondary to primary line ratio for all identified lines. Open symbols - Fe II lines. Mean values of $B_{1}$ were calculated inside the range marked by horizontal bars. $3 \sigma$ error bars are shown.

1.6-2.0 (58 lines) and 2.0-2.6 (14 lines). In Fig. 6 we show the mean value of $A$ and its deviation in each of 4 intervals centered on the middle of the interval. Performing a linear fit of these 4 points forced through the origin, we found the longitudinal field $B_{1}=-54 \pm 12 \mathrm{G}$ (Eq. (4)). This value of the field strength is in good agreement with our direct determinations using the center-of-gravity method and the weighted average magnetic shift (Table 2).

We obtained the magnetic field of about $-60 \div-70 \mathrm{G}$ from measurements of the $\mathrm{H} \alpha$ core $( \pm 0.6 \AA$ from the line center). The same field strength was registered by both the center-ofgravity and the cross-correlation methods. This fact gives independent confirmation of the magnetic field detection.

There are several independent indications that $\chi$ Dra is very likely to possess a weak global magnetic field of about a few tens G. This is the result of direct measurements of line shifts in two pairs of spectra, the behavior of the reduced magnetic shift with effective Lande factors, the different values of the field in the $\mathrm{Fe}$ I and Fe II lines which are distributed in the same spectral region, the firm negative detection in telluric lines also distributed in the same spectral region and the magnetic shift in $\mathrm{H} \alpha$. A more detailed study of this star is beyond the scope of the present work. Further time-resolved spectropolarimetric observations are needed to confirm the positive detection and to ascertain whether the longitudinal magnetic field varies with the star's rotation. The weak field in $\chi$ Dra (as well as possibly in some other stars of our list) would be very important in finding a connection between the upper MS stars which have large-scale organized magnetic fields and $\mathrm{G}, \mathrm{K}, \mathrm{M}$ dwarfs having complex magnetic field structures, in studying a connection between weak magnetic fields in the upper MS stars and weak chemical peculiarities in their atmospheres.

\section{Conclusion}

The first results of the systematic search for magnetic fields in the brightest MS stars show that the accuracy of our magnetic field measurements is sufficient to fulfill the main

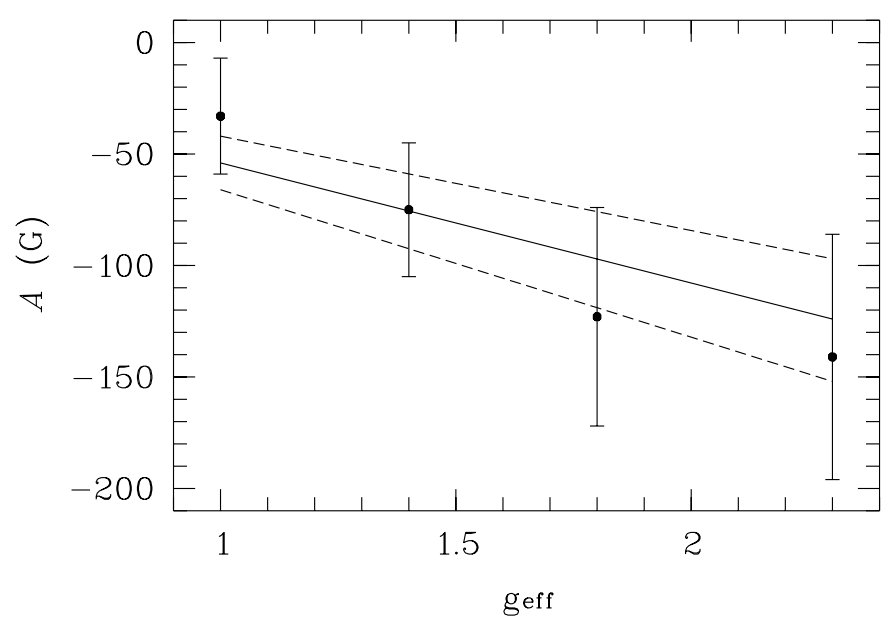

Fig. 6. The reduced magnetic shift in the spectrum of $\chi$ Dra binned in 4 intervals of the effective Lande factors. The error bars correspond to one standard deviation. The best linear fit forced through the origin $\left(A=\left(B_{1} \pm \sigma\right) \cdot g_{\text {eff }}\right)$ is shown.

goal - to survey an unbiased sample of upper MS stars. The accuracy of the measurements ranges from 20 to $300 \mathrm{G}$, depending mainly on spectral type. The spectra in this study were taken under practically identical conditions using the same spectrograph and detector. We are continuing the survey observations. The observations of all targets of the list will help us to derive the selection-free distribution of magnetic fields of upper MS stars.

In some stars we suspect the presence of a weak magnetic field. These suspected stars are the best candidates for more extensive studies and additional observations of these stars are needed. A particular case is the star $\chi$ Dra, where we have a positive detection of a magnetic field. Further observations of $\chi$ Dra are needed to confirm the detection and to ascertain if the field varies with stellar rotation.

Acknowledgements. The authors thank E. A. Barsukova for assistance in performing the observations, J. D. Landstreet and I. I. Romanyuk for useful discussions. We thank the referee, J.-F. Donati, for his helpful critical comments. The VALD database operated in Vienna, Austria and the Simbad database operated at the CDS, Strasbourg, France were used. The work was supported by a grant of the Russian AS for young researchers (Project N. 8) and partly by the RFBR grant N. 01-02-16808.

\section{References}

Allen, C. W. 1977, Astrophysical quantities, third edition (The Athlone Press, University of London)

Andreetta, V., \& Giampapa, M. S. 1995, ApJ, 439, 405

Angel, J. R. P., Borra, E. F., \& Landstreet, J. D. 1981, ApJS, 45, 457

Babcock, H. W. 1958, ApJS, 3, 141

Barker, P. K., Landstreet, J. D., Marlborough, J. M., \& Thompson, I. B. 1985, ApJ, 288, 741

Boesgaard, A. M. 1974, ApJ, 188, 567

Bohlender, D. A., \& Landstreet, J. D. 1990a, MNRAS, 247, 606

Bohlender, D. A., \& Landstreet, J. D. 1990b, ApJ, 358, L25

Borra, E. F., \& Landstreet, J. D. 1973, ApJ, 185, L139

Borra, E. F., \& Landstreet, J. D. 1977, ApJ, 212, 141

Borra, E. F., \& Landstreet, J. D. 1980, ApJS, 42, 421 
Bychkov, B. D., Monin, D. N., Fabrika, S. N., \& Valyavin, G. G. 1997, in Stellar magnetic fields, ed. Yu. V. Glagolevskij, \& I. I. Romanyuk (Moscow), 124

Cramer, N., \& Maeder, A. 1980, A\&A, 88, 135

Donati, J.-F., Semel, M., Rees, D. E., Taylor, K., \& Robinson, R. D. 1990a, A\&A, 232, L1

Donati, J.-F., Semel, M., \& del Toro Iniestia, J. C. 1990b, A\&A, 233, L17

Egret, D., \& Jaschek, M. 1981, in Upper Main Sequence CP Star, 23rd Liège Astrophys. Coll. (Université de Liège), 495

Fabrika, S. N., Shtol', V. G., Valyavin, G. G., \& Bychkov, V. D. 1997, Astron. Lett., 23, 43

Fabrika, S. N., \& Valyavin, G. G. 1998, Bull. Spec. Astrophys. Obs., 45,84

Fabrika, S. N., \& Valyavin, G. G. 1999, in 11th European Workshop on White Dwarfs, ed. S.-E. Solheim, \& E. G. Meistas, ASP Conf. Ser., 169, 214

Glagolevskij, Yu. V., Romanyuk, I. I., Naidenov, I. D., \& Shtol’, V. G. 1989, Astrofiz. Issled. Spec. Astr. Obs., 27, 34

Glagolevskij, Yu. V. 1987, in Magnetic stars, ed. Yu. V. Glagolevskij, \& J. M. Kopylov (Leningrad, "Nauka") 206

Gray, D. F. 1984, ApJ, 277, 640

Hoffleit, D., \& Jaschek, C. 1982, The Bright Star Catalogue (New Haven, Yale University Observatory, 4th revised edition)

Hill, G. M. 1998, MNRAS, 297, 236

Hubrig, S., North, P., \& Mathys, G. 2000, ApJ, 539, 352

Klochkova, V. G., Yermakov, S. V., \& Panchuk, V. E. 1996, Bull. Spec. Astrophys. Obs., 41, 58

Kupka, F., Piskunov, N. E., Ryabchikova, T. A., Stempels, H. C., \& Weiss, W. W. 1999, A\&AS, 138, 119

Landstreet, J. D. 1980, AJ, 85, 611

Landstreet, J. D. 1982, ApJ, 258, 639

Mathys, G., Hubrig, S., Landstreet, J. D., Lanz, T., \& Manfroid, J. 1997, A\&AS, 123, 353

McCook, G. P., \& Sion, E. M. 1999, ApJS, 121, 1

Monin, D. N. 1999, Bull. Spec. Astrophys. Obs., 48, 121
Mukai, K. 1990, PASP, 102, 183

Musaev, F. A. 1996, Astron. Lett., 22, 715

North, P. 1980, A\&A, 82, 230

North, P., \& Cramer, N. 1981, A\&AS, 43, 395

Pierce, A. K., \& Breckinridge, J. B. 1973, KPNO Cont., 559

Piskunov, N. E., Kupka, F., Ryabchikova, T. A., Weiss, W. W., \& Jeffery, C. S. 1995, A\&AS, 112, 525

Preston, G. W. 1971, ApJ, 164, 309

Provin, S. S. 1953, ApJ, 118, 489

Renson, P., Gerbaldi, M., \& Catalano, F. A. 1991, A\&AS, 89, 429

Rice, J. B., \& Wehlay, W. H. 1991, A\&A, 246, 195

Romanyuk, I. I. 2000, in Magnetic fields of chemically peculiar and related stars, ed. Yu. V. Glagolevskij, \& I. I. Romanyuk (Moscow), 18

Ryabchikova, T. A., Piskunov, N. E., Stemples, H. C., Kupka, F., \& Weiss, W. W. 1999, Phys. Scr., 83, 162

Savanov, I. S. 1985, Contrib. Crimea Astrophys. Obs., 73, 92

Schmidt, G. D., \& Smith, P. S. 1995, ApJ, 448, 305

Schoeller, M., Balega, I. I., Balega, Y. Y., et al. 1998, Astron. Lett., 24, 337

Schneider, H. 1994, in The 25th workshop and meeting of european working group on CP stars, ed. I. Jankovics, \& I. J. Vincze (Szombathely, Hungary), 17

Shergin, V. S., Kniazev, A. Yu., \& Lipovetsky, V. A. 1996, Astron. Nachr., 317, 95

Tomkin, J., McAlister, H. A., Hartkopf, W. I., \& Fekel, F. C. 1987, AJ, 93, 1236

Valyavin, G. G., Monin, D. N., Burlakova, T. E., Fabrika, S. N., \& Bychkov, V. D. 1997, in Stellar magnetic fields, ed. Yu. V. Glagolevskij, \& I. I. Romanyuk (Moscow), 128

Valyavin, G. G., \& Fabrika, S. N. 1999, in 11th European Workshop on White Dwarfs, ed. S.-E. Solheim, \& E. G. Meistas, ASP Conf. Ser., 169, 206

Wade, G. A., Donati, J.-F., Landstreet, J. D., \& Mathys, G. 2000, MNRAS, 313, 823 